\title{
The velocity boundary condition at solid walls in rarefied gas simulations
}

\author{
Duncan A. Lockerby \\ Department of Mechanical Engineering, \\ King's College London, London WC2R 2LS, UK \\ Jason M. Reese \\ Department of Mechanical Engineering, \\ Strathclyde University, Glasgow G1 1XJ, UK \\ David R. Emerson and Robert W. Barber \\ Centre for Microfluidics, CLRC Daresbury Laboratory, Warrington WA4 4AD, UK
}

(Dated: October 15, 2003)

\begin{abstract}
Maxwell's original slip boundary condition is widely misapplied in current rarefied gas flow calculations (e.g. in hypersonics, microfluidics). If its commonly-accepted form is applied in simulations of gas flows over curved or moving surfaces, crucial physics can be lost. We give examples of such cases. We also propose a new higher-order boundary condition which is based on Maxwell's original equation and the constitutive relations derived by Burnett. Unlike other higher-order models this is generally applicable to three-dimensional moving surfaces. It is shown that these 'MaxwellBurnett' boundary conditions give much closer agreement with experimental data for Poiseuille flow than existing higher-order models and can also predict Sone's thermal-stress slip flow — a phenomenon which cannot be captured by conventional slip boundary conditions.
\end{abstract}




\section{INTRODUCTION}

In 1879, James Clerk Maxwell published a paper on the viscous stresses arising in rarefied gases [1]. At the time, a reviewer commented that it also might be useful if Maxwell could use his theoretical findings to derive a velocity boundary condition for rarefied gas flows at solid surfaces. Consequently, in an appendix to the paper, Maxwell proposed his now-famous velocity slip boundary condition. This boundary condition was successful in predicting two prior experimental observations: a) that a rarefied gas could slide over a surface [2] and b) that inequalities in temperature could give rise to a force tending to make the gas slide over a surface from colder to hotter regions (which had been discovered by Reynolds, and was known as "thermal transpiration" — now more commonly known as "thermal creep"). What has been subsequently overlooked is the general form of the slip expression derived by Maxwell, and this has some substantial consequences for modern simulations of, e.g., hypersonic aerodynamics and gas flows in microsystems.

Maxwell related the tangential gas velocity slip, $\vec{u}_{\text {slip }}$, to the tangential shear stress, $\vec{\tau}$, and heat flux, $\vec{q}$. This can be written in tensor form so that it is easily applicable to flows over three-dimensional surfaces (a non-tensorial expression can be found in, e.g., Ref. [3]):

$$
\vec{u}_{\text {slip }}=-\frac{(2-\sigma)}{\sigma \mu} \lambda \vec{\tau}-\frac{3}{4} \frac{\operatorname{Pr}(\gamma-1)}{\gamma p} \vec{q},
$$

where $\vec{\tau}=\left(\vec{i}_{n} \cdot \boldsymbol{\Pi}\right) \cdot\left(\mathbf{1}-\vec{i}_{n} \vec{i}_{n}\right), \quad \vec{q}=\vec{Q} \cdot\left(\mathbf{1}-\vec{i}_{n} \vec{i}_{n}\right)$, an arrow denotes a vector quantity, $\sigma$ is the momentum accommodation coefficient (equal to one for surfaces that reflect all incident molecules diffusely, and zero for purely specular reflection), $\mu$ is the gas viscosity at the wall, $\lambda$ is the molecular mean free path at the wall, $\operatorname{Pr}$ is the Prandtl number, $\gamma$ is the specific heat ratio, $p$ is the gas pressure at the wall, $\vec{i}_{n}$ is a unit vector normal and away from the wall, $\boldsymbol{\Pi}$ is the stress tensor at the wall, $\mathbf{1}$ is the identity tensor and $\vec{Q}$ is the heat flux vector at the wall.

If the Navier-Stokes expressions for shear stress and heat flux are adopted, Eq. (1) for a stationary, one-dimensional (planar) wall, can be expressed in the following scalar form:

$$
u_{\mathrm{s}}=\frac{(2-\sigma)}{\sigma} \lambda \frac{\partial u_{x}}{\partial n}+\frac{3}{4} \frac{\mu}{\rho T} \frac{\partial T}{\partial x},
$$

where $n$ is the coordinate normal to the wall, $x$ is the coordinate tangential to the wall, $u_{x}$ is the $x$-component of the gas velocity, $u_{\mathrm{s}}$ is the $x$-component of slip velocity, and $\rho, T$ 
are the density and temperature of the gas at the wall, respectively. It is because of its relative simplicity, compared to Eq. (1), that this expression is remembered as Maxwell's main theoretical result. However, for most surface geometries of practical interest, having curvature and/or wall-normal motion, it is inapplicable and can miss important features of rarefied flow behavior.

In this paper we reassess Maxwell's original equation (1) to examine the effect of implementing the full form of the velocity slip boundary condition in curved or moving geometries, then we propose a new form of higher-order condition which is more accurate at greater degrees of gas rarefaction and is able to capture certain microflow phenomena.

\section{WALL CURVATURE AND MOTION}

For a curved wall in two dimensions, Eq. (1) reduces to

$$
u_{\mathrm{s}}=\frac{(2-\sigma)}{\sigma} \lambda\left(\frac{\partial u_{x}}{\partial n}+\frac{\partial u_{n}}{\partial x}\right)+\frac{3}{4} \frac{\mu}{\rho T} \frac{\partial T}{\partial x},
$$

where $u_{\mathrm{s}}$ is the slip component tangential to the wall, and $u_{n}, u_{x}$ are the gas velocities normal and tangential to the wall, respectively. The additional term that features in Eq. (3) but not in the generally-used Eq. (2) can have a significant influence on the overall velocity slip. For example, if a wall is moving in a direction normal to the surface with a velocity that varies in the tangential direction, then even for flat surfaces (e.g. a deflecting flap) there will be a finite tangential velocity slip. For stationary walls, surface curvature will also give rise to a contribution from the additional term. Einzel, Panzer and Liu [4] derived a boundary condition similar to Eq. (3) for surfaces with curvature. However, their expression did not include the contribution of thermal creep to velocity slip, nor was the relationship to Maxwell's original equation realized. Two examples will show the practical benefit of implementing Maxwell's full formulation.

\section{A. Cylindrical Couette flow}

Recent analytical and molecular dynamics studies [4, 5] suggest that the velocity profile in a rarefied cylindrical Couette flow can become inverted. In the case of a stationary outer cylinder and rotating inner cylinder, 'inverted' means that the radial velocity of the gas becomes greater further away from the moving centre. 


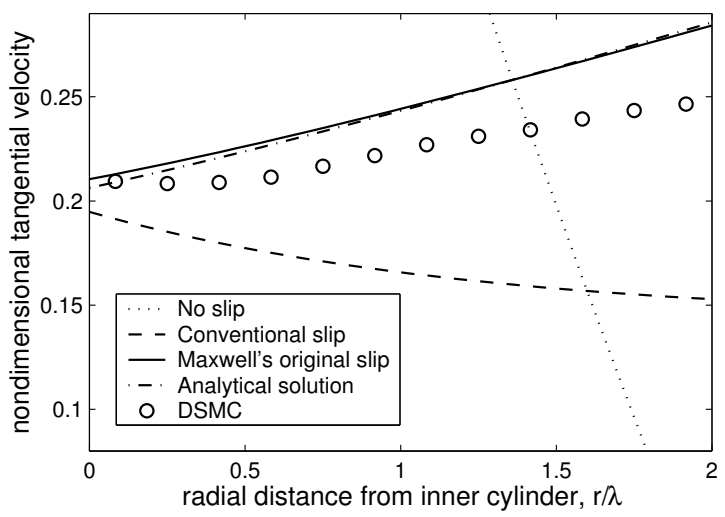

FIG. 1: Nondimensional velocity profiles in cylindrical Couette flow. Comparison of no slip ( . .), conventional slip (--), Maxwell's original slip (-) solutions, an analytical solution [4] $(\cdot-\cdot)$ and DSMC data $[5](\circ)$.

We have performed a simple isothermal calculation using a finite difference discretization of the Navier-Stokes equations to examine the influence of various boundary conditions on the velocity profile. The inner and outer cylinders have radii of $3 \lambda$ and $5 \lambda$, respectively, and the former has a tangential velocity approximately a third of the speed of sound. The gas is argon at STP conditions and the accommodation coefficient, $\sigma$, is 0.1 . Fig. 1 shows a comparison of the velocity profiles (non-dimensionalized by the tangential velocity of the inner cylinder) predicted using the standard no slip condition, the conventional slip condition (Eq.2), Maxwell's original slip condition (Eq.1), DSMC (direct simulation Monte Carlo) molecular dynamics [5], and the analytical method of Einzel, Panzer and Liu [4].

The DSMC method (being a statistical molecular dynamics simulation) is often used as an independent numerical test in the absence of experimental data [6]. That DSMC predicts an inverted velocity profile is strong corroborative evidence that the phenomenon exists. The conventional slip condition, Eq. (2), evidently cannot predict such behavior, however Maxwell's original slip condition, Eq. (1), produces a velocity profile inversion. Close quantitative agreement between DSMC and simulation is not expected as the degree of gas rarefaction in this problem means that continuum fluid models are at the limit of their applicability. 


\section{B. Drag on an unconfined sphere}

Isothermal slip flow past an unconfined sphere at very low Reynolds and Mach numbers was first analyzed by Basset [7] using Stokes' creeping flow approximation. The effect of slip was incorporated into the analysis using a velocity boundary condition for isothermal flows of an equivalent form to Maxwell's original boundary condition, Eq. (1). Basset's analysis showed that the skin friction drag, $D_{s}$, on an unconfined sphere of radius $a$, in a flow stream of velocity $U$, can be written as:

$$
D_{s}=4 \pi \mu U a^{2}\left(\frac{\sigma}{\sigma(a-3 \lambda)+6 \lambda}\right) \text {. }
$$

However, if the conventional form of Maxwell's boundary condition, Eq. (2), is used in the derivation a different expression for the skin friction drag is obtained:

$$
D_{s}=4 \pi \mu U a\left(\frac{\sigma(a+\lambda)-2 \lambda}{\sigma(a-2 \lambda)+4 \lambda}\right) .
$$

The disparity between the two drag predictions is due to the omittance of curvature in the conventional boundary condition. In the limiting case of a perfectly smooth sphere, such that all incident molecules are reflected specularly $(\sigma=0)$, there is no means by which the wall can transfer tangential momentum to or from the gas. Therefore, the drag due to skin-friction should be zero and, indeed, we find that Basset's drag equation predicts no skin friction. However, Eq. (5) predicts a finite value of negative skin friction drag (i.e. a thrust). This non-physical prediction demonstrates the importance of employing the original, as opposed to the conventional, form of Maxwell's boundary condition for curved surfaces.

\section{HIGHER-ORDER BOUNDARY CONDITIONS}

In wall-dominated flows, such as those typical of microfluidics, the accuracy of the overall numerical solution is highly dependent on the accuracy of the boundary conditions. This sensitivity is such that several attempts have been made to derive boundary conditions of second or higher spatial order [8-12]. However, even for simple flows, there has been no general consensus as to the exact form these should take.

While there is no commitment made to the form of the shear stress tensor or heat flux vector in Maxwell's original condition, Eq. (1), normally the Navier-Stokes constitutive relations are assumed. This leads us to propose in this paper that, instead, higher-order constitutive 
relations (appropriate for rarefied flows) are employed. These yield higher-order boundary conditions automatically that will also be applicable to flows over three-dimensional non-stationary surfaces, unlike previous higher-order slip boundary conditions.

One such higher-order set of constitutive relations is the Burnett equations, derived from the terms up to second-order in a series solution in Knudsen number to the Boltzmann equation [13]. Although their complexity and nonlinearity makes them difficult to solve numerically [14], their assumed applicability to rarefied, high Knudsen number flows can be exploited within Maxwell's boundary condition without having to solve the tensor expressions for the entire flow field. In flows dominated by gas-surface interactions, there is also justification in adopting a more accurate model at the boundaries (Burnett) than the flow itself (Navier-Stokes).

The complete two-dimensional form of this equation is lengthy, so attention is restricted here to the linear higher-order terms only, which makes our analysis applicable to weak variations in flow variables only (the full nonlinear form of the Burnett stress tensor and heat flux vector are given in Ref. [13]). For flow over a two-dimensional wall, what we term as the "linearized Maxwell-Burnett boundary condition" is as follows:

$$
\begin{aligned}
u_{\mathrm{s}}= & \frac{(2-\sigma)}{\sigma} \lambda\left(\frac{\partial u_{x}}{\partial n}+\frac{\partial u_{n}}{\partial x}\right)+\frac{3}{4} \frac{\mu}{\rho T} \frac{\partial T}{\partial x} \\
& +\frac{(2-\sigma)}{\sigma} \lambda\left(2 \frac{\mu}{\rho^{2}} \frac{\partial^{2} \rho}{\partial x \partial n}-\frac{\mu}{\rho T} \frac{\partial^{2} T}{\partial x \partial n}\right) \\
& +\frac{3}{16 \pi} \frac{\operatorname{Pr}(\gamma-1)}{\gamma} \lambda^{2}\left[(45 \gamma-61) \frac{\partial^{2} u_{x}}{\partial x^{2}}\right. \\
& \left.+(45 \gamma-49) \frac{\partial^{2} u_{n}}{\partial x \partial n}-12 \frac{\partial^{2} u_{x}}{\partial n^{2}}\right]
\end{aligned}
$$

which is formally second-order in space. We now show the effect this form of the boundary condition has in two fundamental configurations.

\section{A. Plane Poiseuille Flow}

For plane Poiseuille flow, second-order slip boundary conditions have the general form:

$$
u_{\mathrm{s}}=A_{1} \lambda \frac{\partial u_{x}}{\partial n}-A_{2} \lambda^{2} \frac{\partial^{2} u_{x}}{\partial n^{2}} .
$$

As yet, no consensus has been reached on the correct value of the coefficient $A_{2}$ since theoretical predictions have not compared well to experimental observations. There is, in 
TABLE I: Values of $A_{2}$ in Eq. (7).

\begin{tabular}{|c|c|c|c|}
\hline Reference & Study & Molecule & $A_{2}$ \\
\hline Lang $[10]$ & theoretical & monatomic & 1.43 \\
\hline Schamberg [11] & theoretical & monatomic & 1.31 \\
\hline Deissler [12] & theoretical & $\begin{array}{l}\text { monatomic } \\
\& \text { diatomic }\end{array}$ & 1.125 \\
\hline Cercigani $[8]$ & theoretical & monatomic & 0.98 \\
\hline Maurer et al. [15] & experimental & $\begin{array}{l}\text { Nitrogen } \\
\text { Helium }\end{array}$ & $\begin{array}{l}0.26 \pm 0.1 \\
0.23 \pm 0.1\end{array}$ \\
\hline $\begin{array}{c}\text { Maxwell-Burnett } \\
\text { Eq. (6) }\end{array}$ & theoretical & $\begin{array}{c}\text { monatomic } \\
\text { diatomic }\end{array}$ & $\begin{array}{c}0.19 \\
0.145\end{array}$ \\
\hline Sreekanth [16] & experimental & Nitrogen & $0.14^{a}$ \\
\hline $\begin{array}{l}\text { Karniadakis } \\
\& \text { Beskok [9] }\end{array}$ & theoretical & $\begin{array}{l}\text { monatomic } \\
\& \text { diatomic }\end{array}$ & -0.5 \\
\hline
\end{tabular}

${ }^{a}$ Data for cylindrical Poiseuille flow.

any case, some evidence to indicate that both $A_{1}$ and $A_{2}$ are geometry-dependent. Table I compares theoretical and experimental values proposed for $A_{2}$ for channel flow, as well as the value of this coefficient in the $\lambda^{2} \partial^{2} u_{x} / \partial n^{2}$ term in the Maxwell-Burnett boundary condition, Eq. (6). A value for a diatomic gas in this case has been estimated by assuming that the translational heat flux dominates the rotational and vibrational modes. It is clear that our Maxwell-Burnett condition provides much closer agreement with the limited experimental data than other higher-order boundary models. Its geometry-dependence will be explored in future papers.

\section{B. Thermal-stress slip flow}

Thermal-stress slip flow is a rarefaction phenomenon that was originally predicted by Sone [17]. Via an asymptotic analysis of the Boltzmann equation, he showed that a tangential variation in the wall-normal temperature gradient could induce velocity slip. This is distinct from thermal creep, and cannot be captured by either the conventional slip equation, Eq. (2), or Maxwell's original boundary condition with Navier-Stokes expressions for the shear stress 
and heat flux terms.

Sone's configuration is a gas (initially stationary) between two stationary non-coaxial cylinders of different uniform temperature, $T_{1}$ and $T_{2}$. In the absence of thermal creep (i.e. the boundary temperature jump is not considered) no conventional boundary condition has the mechanism to predict a slip flow. Sone, however, calculated the slip-flow field as shown by the streamlines and directional arrows in Fig. 2 a). The outer cylinder is held at a higher temperature and this generates a steady clockwise circulation in the gas (and anti-clockwise when $T_{1}>T_{2}$ ).

For a similar cylinder configuration, but using a finite volume code to solve the NavierStokes equations with the linearized Maxwell-Burnett boundary condition, Eq. (6) (nonlinear effects are negligible as the temperature difference is small), the steady flow pattern is shown in Fig. 2 b). It is clear that the phenomenon predicted by Sone is captured in our simulation due to the inclusion of the new form of the boundary condition. Independent experimental verification is now needed.

For a stationary gas, Eq. (6) reduces to a single term:

$$
u_{\mathrm{s}}=-\frac{(2-\sigma)}{\sigma} \lambda\left(\frac{\mu}{\rho T} \frac{\partial^{2} T}{\partial x \partial n}\right) .
$$

It is this term that is responsible for initiating the thermal-stress slip flow in Fig. 2. Interestingly, this term also featured in Maxwell's original derivation, though its presence has been forgotten over time.

\section{DISCUSSION}

The simplified version of Maxwell's equation, passed down through successive research generations, is not applicable to the two and three-dimensional boundaries typical of rarefied gas flows in complex geometries. If Maxwell's velocity slip equation is to be used then care should be taken in adopting the full form of Maxwell's original equation, Eq. (1), which relates tangential velocity slip to tangential shear stress and heat flux.

Compared to other higher-order boundary conditions derived from kinetic theory, the Maxwell-Burnett boundary condition we propose is simplistic. Nevertheless, it has been shown both to agree closely with experimental Poiseuille flow data and to predict the phenomenon of thermal-stress slip flow. It is straightforwardly applicable to three-dimensional 

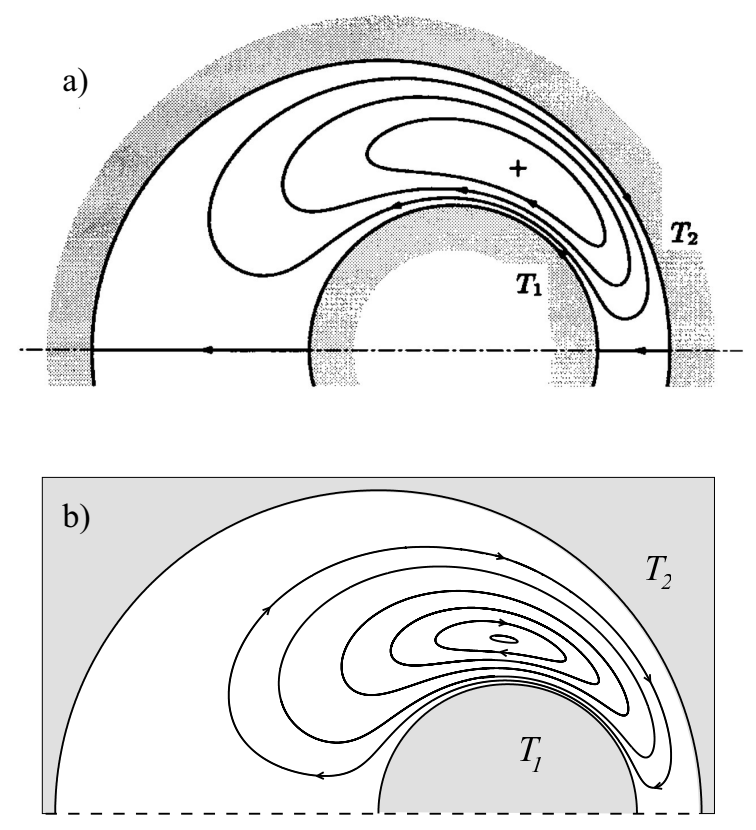

FIG. 2: Streamlines of thermal-stress slip flow between non-coaxial cylinders (uniform temperatures, $\left.T_{2}>T_{1}\right)$; a) solution of the Boltzmann equation reproduced from [18], b) finite volume solution using the Maxwell-Burnett boundary condition.

moving surfaces, and can also be used for diatomic gas molecules if appropriate changes are made to the Burnett coefficients.

\section{Acknowledgments}

The authors are grateful to the Leverhulme Trust (UK) and the Medical Research Council's MicroFAST programme (grant reference 57719) for supporting this research financially.

[1] J. C. Maxwell, Phil. Trans. Roy. Soc., Part 1 170, 231 (1879).

[2] A. Kundt and E. Warburg, Pogg. Ann. clv, 337 (1875).

[3] M. Gad-el-Hak, ASME J. Fluid Eng. 121, 5 (1999).

[4] D. Einzel, P. Panzer, and M. Liu, Phys. Rev. Lett. 64, 2269 (1990).

[5] K. W. Tibbs, F. Baras, and A. L. Garcia, Phys. Rev. E 56, 2282 (1997).

[6] J. M. Reese, M. Gallis, and D. A. Lockerby, Phil. Trans. Roy. Soc. (to be published).

[7] A. B. Basset, A treatise on hydrodynamics (Deighton, Bell and Co., Cambridge, 1888). 
[8] C. Cercignani, Mathematical methods in kinetic theory (Plenum, New York, 1969).

[9] G. E. Karniadakis and A. Beskok, Micro Flows: Fundamentals and Simulation (Springer, 2002).

[10] H. Lang, Phys. Fluids 19, 366 (1976).

[11] R. Schamberg, Ph.D. thesis, California Institute of Technology (1947).

[12] R. Deissler, Int. J. Heat Mass Transfer 7, 681 (1964).

[13] S. Chapman and T. G. Cowling, The Mathematical Theory of Non-Uniform Gases (Cambridge University Press, 1970), 2nd ed.

[14] X. L. Zhong, R. W. MacCormack, and D. R. Chapman, AIAA J. 31, 1036 (1993).

[15] J. Maurer, P. Tabeling, P. Joseph, and H. Willaime, Phys. Fluids 15, 2613 (2003).

[16] A. Sreekanth, in Proceedings of the Sixth International Symposium on Rarefied Gas Dynamics (Academic Press, New York, 1969), p. 667.

[17] Y. Sone, Phys. Fluids 15, 1418 (1972).

[18] Y. Sone, Ann. Rev. Fluid Mech. 32, 779 (2000). 